\title{
Study of EDC/NHS immobilization for plumbous detection using surface plasmon resonance
}

\begin{abstract}
The presence of plumbous ( $\mathrm{Pb} 2+)$ in Irrigation water is harmful for the environment as well as human health. Herein, a simple yet effective sensor for $\mathrm{Pb} 2+$ detection is presented utilizing a surface plasmon resonance technique. The proposed sensor consists of a combination of 1-ethyl-3-(3-dimethylaminopropyl) carbodiimide hydrochloride (EDC) and N-hydroxysuccinimide (NHS) were attached to a gold layer offers a new option for heavy metal detection. The EDC/NHS works as a sensing layer that able to detect $\mathrm{Pb} 2+$ down to 15 ppm that matches with the United States Environment Protection Agency.
\end{abstract}

Keyword: Heavy metal; Plumbous detection; Surface plasmon 